\title{
A Modified Structure for All-Glass Photonic Bandgap Fibers: Dispersion Characteristics and Confinement Loss Analysis
}

\author{
Sanjaykumar Gowre, ${ }^{1}$ Sudipta Mahapatra, ${ }^{1}$ and P. K. Sahu ${ }^{2}$ \\ ${ }^{1}$ Department of Electronics \& Electrical Communication Engineering, Indian Institute of Technology Kharagpur, Kharagpur, \\ West Bengal 721302, India \\ ${ }^{2}$ School of Electrical Sciences, Indian Institute of Technology Bhubaneswar, Bhubaneswar, Odisha 751013, India
}

Correspondence should be addressed to Sanjaykumar Gowre; sanjaygowre@gmail.com

Received 7 June 2013; Accepted 2 July 2013

Academic Editors: Y. S. Kivshar and S. R. Restaino

Copyright (c) 2013 Sanjaykumar Gowre et al. This is an open access article distributed under the Creative Commons Attribution License, which permits unrestricted use, distribution, and reproduction in any medium, provided the original work is properly cited.

This paper investigates a modified structure for all-glass photonic bandgap fiber (AGPBF) having up-doped silica rods in the cladding region instead of air holes using plane wave expansion (PWE) and finite difference time domain (FDTD) methods. The proposed AGPBF structure exhibits tunable dispersion properties and improved confinement loss. It is observed that the confinement loss can be reduced simply by using a higher doping concentration in silica rods in the cladding. Also, it is possible to achieve flattened dispersion of the order of $1 \mathrm{ps} / \mathrm{nm} / \mathrm{km}$ over a wide wavelength range.

\section{Introduction}

Photonic bandgap fibers (PBFs) have the most basic and original structure in the large family of photonic crystal fibers (PCFs) $[1,2]$, as the light in these structures is confined in a low index core compared to all the other fibers. In PBF, light is confined to the core by the photonic bandgap effect $(\mathrm{PBG})$, instead of total internal reflection. Hollow-core PBF (HC-PBF) with air holes in a glass background allows lowloss propagation in an empty core, giving the fibers several interesting properties and applications [3]. Since much of the optical power in PBFs can travel in air and not in the glass, they do not suffer from the same limitations to loss as conventional fibers and can exhibit radically reduced optical nonlinearity, making them promising candidates for future ultralow loss transmission fibers [4].

The fabrication of the hollow core photonic bandgap fiber with required specification is difficult due to air-hole deformation and measured losses are more. In order to decrease the confinement loss, we need to accommodate more number of air holes in the given cladding region. Further, to satisfy this condition, the interhole spacing should be minimum, which causes the merger of the adjacent air holes.
Confinement loss in triangular air-guiding PBFs has been discussed in [5], and it is concluded that, with $d / \Lambda=0.9$ ( $d$ and $\Lambda$ are the air-hole diameter and interhole spacing, resp.), at least 20 rings of air holes that are totally 1380 air holes are required to reduce the confinement loss to a level of $0.01 \mathrm{~dB} / \mathrm{km}$. Confinement loss in air-core PBF having 8 rings of (totally 264) circular air holes has been analyzed, and the confinement loss can be reduced to lower than $0.01 \mathrm{~dB} / \mathrm{km}$ with $d / \Lambda=0.97$ [6]. However, it is very difficult to make aircore PBF with so many air holes in the former case and so high $d / \Lambda$ for circular air holes in the latter case, respectively.

Confinement loss can be reduced by increasing the airfilling fraction of PBF; the air-filling fractions in [5] is $73 \%$, whereas, in order to increase air-filling fraction further, cobweb designs are used to reduce confinement loss in PCF, which can have very high air-filling fractions. Cobweb aircore PBF with air-filling fraction of $94 \%$ has been reported in $[5,6]$. However, large air-filling fraction may cause splicing problems, and Fresnel refection at the end facet may make it difficult to integrate such air-core PBF with conventional fibers.

In conventional optical fiber, the group velocity dispersion of the guided mode is dominated by the dispersion 
of the bulk material from which the core is made. Instead, due to a negligible contribution from core material, the total dispersion in PBF is completely dominated by waveguide dispersion. Since the PBF can only confine light of wavelengths within this bandgap, their transmission spectrum can be thought of as one of the transparent material with a transmission window expanding the bandgap width.

In this paper, we present a large core all-glass photonic bandgap fiber structure having up-doped silica rods in the cladding region. This arrangement will avoid the problems of air-hole deformation, and we can accommodate 118 doped silica rods in the cladding region in order to reduce the confinement loss. Further, the proposed all-glass photonic bandgap fiber (AGPBF) structure is analyzed for tunable dispersion properties, and the confinement loss is estimated by plane wave expansion (PWE) method.

The organization of the paper is as follows. Section 2, following the introduction, briefly presents the design of the all-glass PBF. Section 3 compiles flattened dispersion and dispersion shifted fiber results including the confinement loss analysis to show the superiority of the proposed design, and Section 4 concludes the paper.

\section{All-Glass PBF Design}

The main objective of the work reported in this paper is to overcome the limitations of conventional photonic bandgap fibers like air-hole deformation and emergence of additional air holes during the fabrication of the fiber [7].

2.1. Effect of the Germanium Doping on Refractive Index. Predicting the refractive index, material dispersion, and profile dispersion for various $\mathrm{GeO}_{2}$ doped silica fibers is vital for finding the optimum dispersion-shifted and dispersionflattened fiber designs in the minimum loss $1.55 \mu \mathrm{m}$ wavelength region. Addition of $\mathrm{GeO}_{2}$ increases the refractive index. The variation of the refractive index of doped silica with $\mathrm{GeO}_{2}$ doping is empirically obtained as [8]

$$
n=\sqrt{\sum_{i=1}^{3} \frac{A_{i} B_{i} f \lambda^{2}}{\lambda^{2}-z_{i}^{2}}},
$$

where $n$ is the refractive index and $\lambda$ is the vacuum wavelength. The summation is over three oscillators where $A_{i}$ and $B_{i}$ are the oscillator strength, $z_{i}$ is the oscillator wavelength, and $f$ is the doping concentration. Table 1 gives the values of $A_{i}, B_{i}$, and $z_{i}$.

Figure 1 shows the variation in the refractive index as a function of the doping concentration. It is observed that, as the doping concentration is increased from 0 to $13.5 \%$, the refractive index increases. In our proposed all-glass photonic bandgap fiber, germanium doped rods are used in the cladding region.

The proposed all-glass photonic bandgap fiber (AGPBF) structure is shown in Figure 2, in which the cladding region consists of a triangular array of 118 up-doped silica rods embedded in a cylindrical structure made up of pure silica. The proposed AGPBF structure can be fabricated by the
TABle 1: Parameter values for $\mathrm{GeO}_{2}$ doping.

\begin{tabular}{lcc}
\hline S. no. & Parameter & Values \\
\hline 1 & $A_{1}$ & 0.2045154578 \\
2 & $A_{2}$ & 0.06451676258 \\
3 & $A_{3}$ & 0.1311583151 \\
4 & $B_{1}$ & -0.1011783769 \\
5 & $B_{2}$ & 0.1778934999 \\
6 & $B_{3}$ & -0.1064179581 \\
7 & $z_{1}$ & 0.06130807320 \\
8 & $z_{2}$ & 0.1108859848 \\
9 & $z_{3}$ & 8.964441861 \\
\hline
\end{tabular}

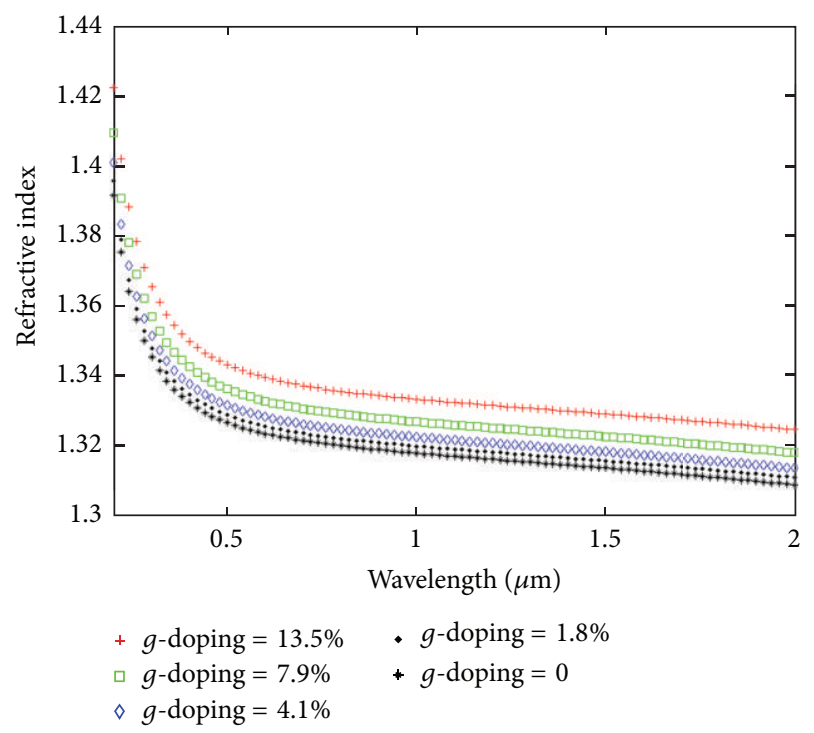

FIGURE 1: Effect of the germanium on the refractive index.

conventional stack and draw process used for photonic crystal fibers. The silica rods used in the cladding structure are composed of up-doped silica and may be fabricated by the modified chemical vapour deposition (MCVD) process, while the core region is simply formed from a pure silica rod. Finally, by milling the circular rods into a hexagonal shape, we may stack a completely solid preform and thereby eliminate the use of air holes which may introduce both transverse and longitudinal inhomogeneities in the lattice structure during fabrication. We have chosen this structure of AGPBF as the triangular lattice of high-index rods provides the largest bandgap for a given index contrast [9].

The core region of the fibre has been created by omitting a high-index rod in the centre of the fibre and, therefore, is of pure silica. The proposed structure has an additional design parameter in terms of doping concentration in addition to the diameter of the silica rods, pitch, and refractive index. The mode field distribution of the proposed all-glass PBF is depicted in Figure 3, and it is evident from the figure that the guided mode is well confined. 


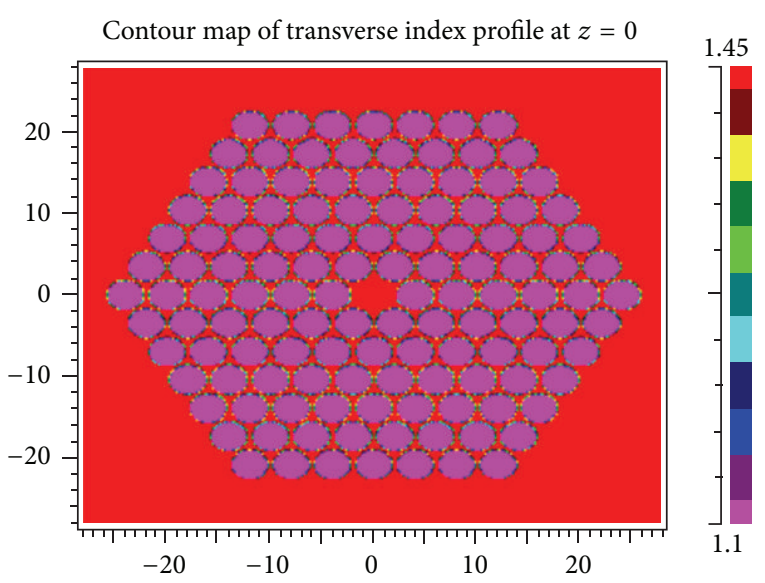

FIGURE 2: Proposed all-glass photonic bandgap fiber.
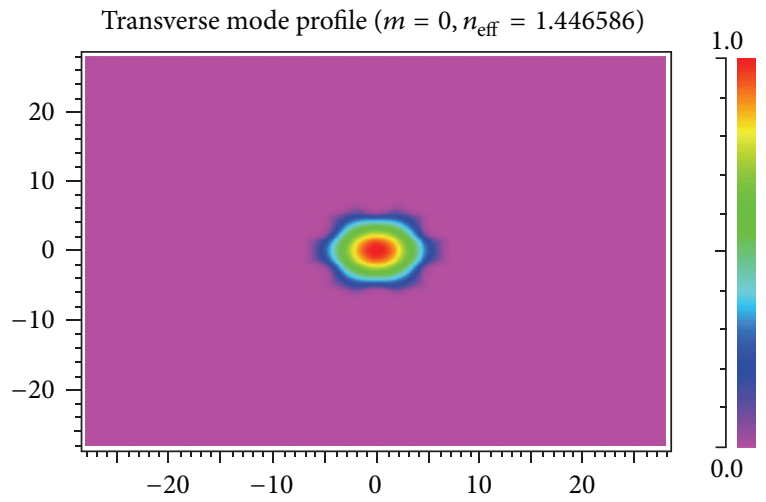

FIgURE 3: Transversal field intensity distribution at a wavelength of $1.55 \mu \mathrm{m}$ for the fundamental guiding mode.

\section{Results and Discussions}

3.1. Confinement Loss. The main reasons for the occurrence of confinement loss in HC-PCF are due to the finite number of rows of the air holes and the width of the bandgap. Consequently, finding a lattice that can be fabricated and exhibits the largest bandgap is of paramount importance. To date, the triangular lattice, which is a circular array of air holes packed in a triangular arrangement, is found to be the most promising, as its fabrication is relatively easy. Therefore, it is necessary to consider the confinement loss in the analysis of both solid-core (AHPCF) and hollow-core PCFs (PBF). As a consequence, all the PCF guided modes are leaky. For example, in solid-core PCFs, light is confined within a core region by the air holes. Light will move away from the core if the confinement provided by the air-holes is inadequate. This means that it is important to design such aspects of the PCF structure, as air-hole diameter $(d)$ and hole-to-hole spacing $(\Lambda)$, in order to realize low-loss PCFs. In particular, the ratio between the air-hole diameter and the pitch must be designed to be large enough to confine light into the core. On the other hand, a large value of the ratio makes the PCF multimode.

The numerical investigation of the proposed AGPBF structure indicates that, by keeping the ratio of $(d / \Lambda)$ large

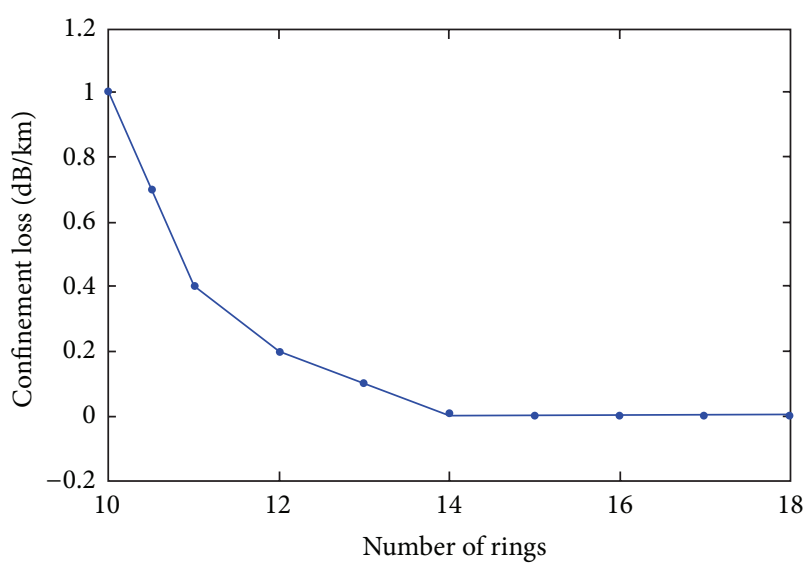

FIGURE 4: Confinement loss as a function of number of rings.

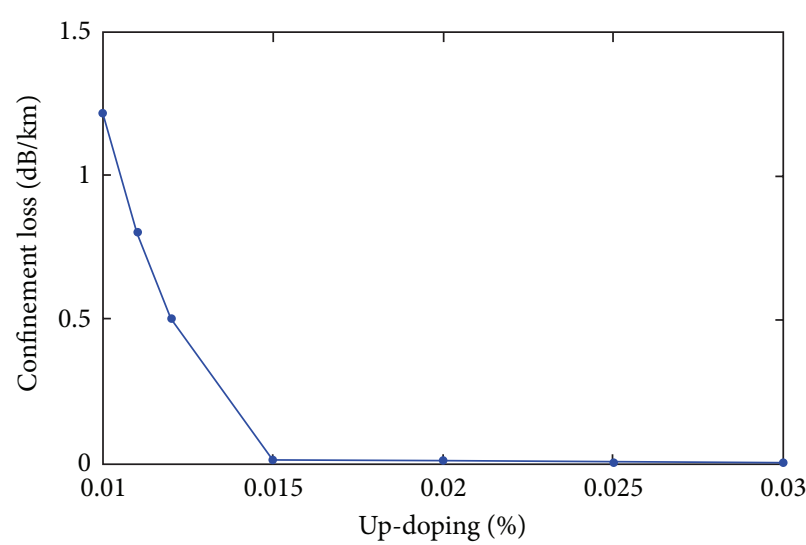

FIgURE 5: Confinement loss as a function of up-doping.

enough, it is possible to confine the light into the core region. The effect of the number of rings, up-doping, variation in interrod spacing $(\Lambda)$, and rod diameter $(d)$ on the confinement loss is analyzed with the help of a finite difference time domain (FDTD) method. Figure 4 shows confinement loss variation with the number of up-doped rings. Results indicate that a larger number of rings of rods provide more confined guided modes and result in lower confinement loss. Although the confinement loss can be reduced simply by increasing the number of rings, this will result in a thicker fiber diameter.

Figure 5 shows the variation of the confinement loss with the doping concentration, and by comparing Figure 4 and Figure 5 we can verify the fact that confinement loss can be reduced by increasing the up-doping level instead of increasing the number of rings.

3.2. Dispersion Flattened Fiber. The dispersion properties of conventional single mode fiber are receiving a lot of research interest in connection with optical communication, soliton propagation, and controlling nonlinear effects. Currently, the dispersion characteristics of PBFs are being investigated by 
many of the researchers working in this area [9-11]. The dispersion encountered in PBF is calculated as follows:

$$
D=-\frac{\lambda}{c} \frac{d^{2} n}{d \lambda^{2}}
$$

where $D$ is the group velocity dispersion (GVD) in $\mathrm{ps} / \mathrm{nm} / \mathrm{km}, n$ is the refractive index, and $\lambda$ is the operating wavelength. PBFs offer very high anomalous waveguide dispersion in a large wavelength range where the fiber operates in single mode regime.

In order to accurately determine the dispersion properties of the proposed AGPBF, we employed the plane-wave expansion method, but, to include also the material dispersion of silica, we used an iterative scheme incorporating the generally used Sellmeier dispersion relation. The result of such a dispersion calculation for a triangular AGPBF with $d$ values ranging from $5 \mu \mathrm{m}$ to $8 \mu \mathrm{m}$ is illustrated in Figure 6 . For applications within the area of telecommunication, we have focused on the wavelength range from $1.55 \mu \mathrm{m}$ to $1.7 \mu \mathrm{m}$ with $d=6 \mu \mathrm{m}, \Lambda=8 \mu \mathrm{m}, \mathrm{Nr}=6$, and refractive index contrast between core and cladding $=2 \%$. It can be observed from the result that, as silica rod diameter is increased from $5 \mu \mathrm{m}$ to $8 \mu \mathrm{m}$, the dispersion exhibited is more flattened.

3.3. Dispersion Shifted Fiber. Standard telecom fibers exhibit zero chromatic dispersion in the $1.31 \mu \mathrm{m}$ wavelength region. This was convenient for early optical fiber communications systems, which often operated around $1310 \mathrm{~nm}$. However, the $1.55 \mu \mathrm{m}$ region later became more significant, because the fiber losses are lower there, and erbium-doped fiber amplifiers (EDFAs) are available for this region. In this wavelength region, however, standard single-mode fibers exhibit significant anomalous dispersion. For linear transmission, this can be a problem, because it leads to significant dispersive pulse broadening, thereby limiting the achievable transmission rates or distance. Therefore, the so-called dispersion-shifted fibers have been developed, which have modified waveguide dispersion so as to shift the zero dispersion wavelength into the $1.55 \mu \mathrm{m}$ region. This is achieved by modifying the refractive index profile of the core. Common index profiles of dispersion-shifted fibers have a triangular, trapezoidal, or Gaussian shape.

The overall GVD has two contributions, the material dispersion and the waveguide dispersion. However, for a given wavelength and material, the design of a simple SIF has two degrees of freedom, the core radius and the index difference between the core and cladding. For example, one can shift the zero of GVD from $1.31 \mu \mathrm{m}$ to $1.55 \mu \mathrm{m}$ by increasing the index difference between core and cladding and reducing the core size.

For photonic bandgap fibers with small mode areas, which can exhibit particularly strong waveguide dispersion, the zero dispersion wavelength (ZDW) can be shifted into the visible spectral region. Photonic bandgap fibers as well as some other fiber designs can exhibit two or even three different zero dispersion wavelengths. Operation of a telecom system around the zero dispersion wavelength greatly reduces dispersive broadening. At the same time, however, the signals

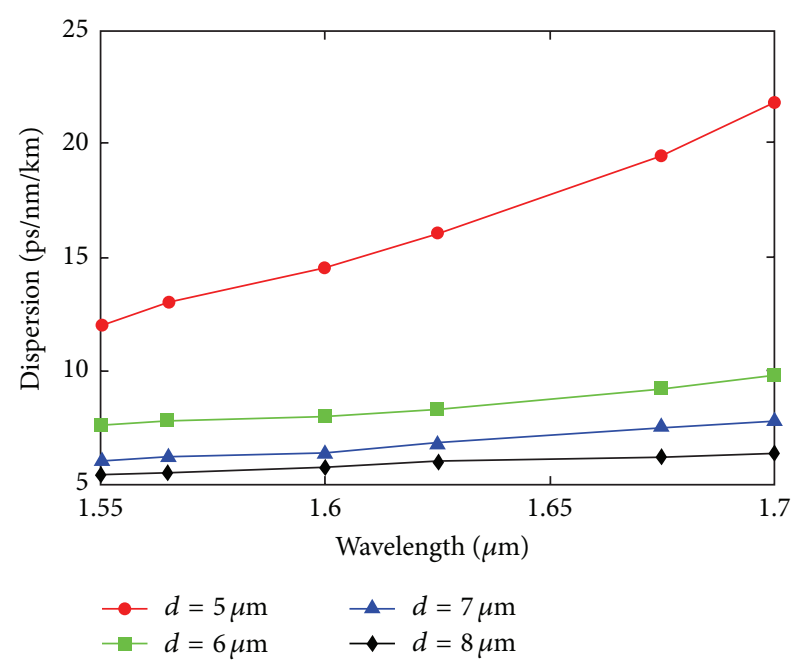

FIGURE 6: Dispersion characteristics of proposed all-glass PBF.

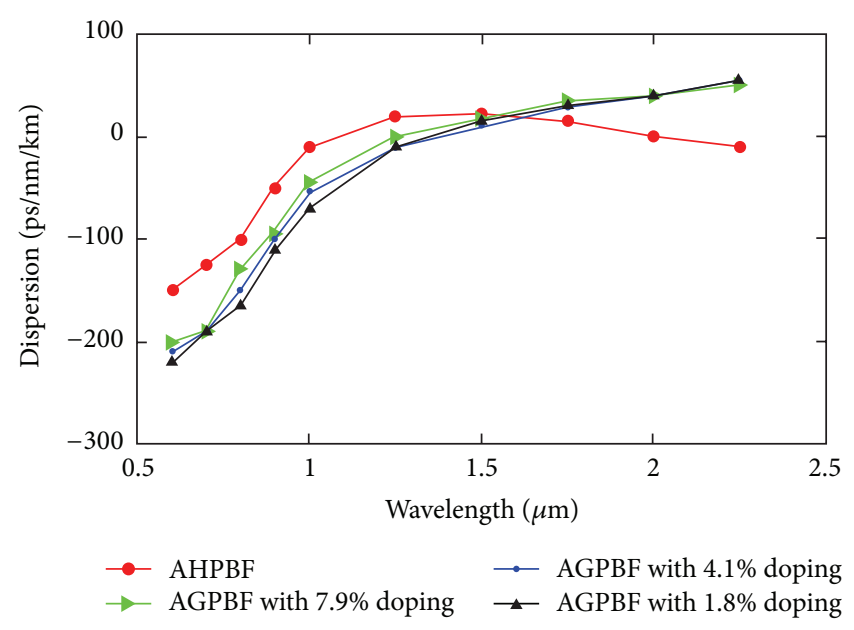

FIgURE 7: Effect of doping concentration on the dispersion.

become relatively sensitive to optical nonlinearities of the fiber, such as four-wave mixing, which can be phase matched under these conditions. It is therefore not always advantageous to operate in this regime; an improved approach is dispersion management in the form of alternatively using fibers with different dispersions.

To overcome the problem of varying either rod diameter (d) or interrod spacing $(\Lambda)$ or the ratio of $d / \Lambda$ to shift the zero dispersion wavelength (ZDW) to any desired value, we have proposed an all-glass PBF structure with interrod spacing $(\Lambda)=2.3 \mu \mathrm{m}$, refractive index of the core $\left(n_{\mathrm{co}}\right)=1.45$, and number of rings $=06$. The diameter of the up-doped silica rod is $1.0 \mu \mathrm{m}$. In our proposed structure of AGPBF, the ZDW can be shifted to any wavelength by the variation in the doping concentration.

Figure 7 shows the effect of varying the doping concentration on the dispersion. For conventional PBF, the zero dispersion is at $1.08 \mu \mathrm{m}$, and the zero dispersion wavelength is shifted to lower wavelength values as the doping concentration is increased. From Figure 7, the ZDW is at 
$1.4 \mu \mathrm{m}$ for doping concentration of $1.8 \%, 1.38 \mu \mathrm{m}$ for doping concentration of $4.1 \%$, and $1.22 \mu \mathrm{m}$ for doping concentration of $7.9 \%$.

\section{Conclusions}

In this paper, we have investigated the dispersion and confinement loss in an all-glass photonic bandgap fiber made solely from a combination of pure silica and up-doped silica rods. This fibre design is interesting, because it allows us to obtain some of the unique optical properties of PBF (like flattened dispersion and dispersion shifting), while having a fibre that is relatively easy to manufacture due to the elimination of air holes in the cladding region. The proposed AGPBF has large positive waveguide dispersion. We have shown that this unique property could be used to shift the zero dispersion wavelength down to $1220 \mathrm{~nm}$. Results indicate that, for large number of rods in the cladding region and with more doping concentration, the confinement loss is very low in our proposed structure. Further, it is observed from the results that, as rod diameter is increased from $5 \mu \mathrm{m}$ to $8 \mu \mathrm{m}$, the dispersion gets flattened, and for rod diameter of $8 \mu \mathrm{m}$ the dispersion is of the order of $1 \mathrm{ps} / \mathrm{nm} / \mathrm{km}$ over a wavelength range from $1550 \mathrm{~nm}$ to $1700 \mathrm{~nm}$. Moreover, by varying the doping concentration, it is possible to shift the zero dispersion wavelength to any desired value, while keeping rod diameter, interrod spacing, and the ratio of rod diameter to interrod spacing constant.

\section{Acknowledgment}

This work is supported by Visvesvaraya Technological University, Belgaum, Karnataka State, under Award no. VTU/Aca/2009-10/A-9/11621.

\section{References}

[1] J. C. Knight, J. Broeng, T. A. Birks, and P. S. J. Russell, "Photonic band gap guidance in optical fibers," Science, vol. 282, no. 5393, pp. 1476-1478, 1998.

[2] R. F. Cregan, B. J. Mangan, J. C. Knight et al., "Single-mode photonic band gap guidance of light in air," Science, vol. 285, no. 5433, pp. 1537-1539, 1999.

[3] G. Bouwmans, F. Luan, J. C. Knight et al., "Properties of a hollow-core photonic bandgap fiber at $850 \mathrm{~nm}$ wavelength," Optics Express, vol. 11, no. 14, pp. 1613-1620, 2003.

[4] M. S. Charlene, N. Venkataraman, T. G. Michael et al., "Lowloss hollow-core silica/air photonic bandgap fibre," Nature, vol. 424, no. 6949, pp. 657-659, 2003.

[5] K. Saitoh and M. Koshiba, "Confinement losses in air-guiding photonic bandgap fibers," IEEE Photonics Technology Letters, vol. 15, no. 2, pp. 236-238, 2003.

[6] Y. Xu and A. Yariv, "Loss analysis of air-core photonic crystal fibers," Optics Letters, vol. 28, no. 20, pp. 1885-1887, 2003.

[7] D. Pysz, R. Stępień, P. Szarniak, R. Buczyński, and T. Szoplik, "Comparing characteristics and prospects of fabrication of multicomponent photonic crystal fibres with different lattice structures," in Proceedings of the SPIE, Lightguides and Their Applications II, (TAL '03), vol. 5576, pp. 74-80, October 2003.
[8] H. R. D. Sunak and S. P. Bastien, "Refractive index and material dispersion interpolation of doped silica in the 0.6$1.8 \mu \mathrm{m}$ wavelength region," IEEE Photonics Technology Letters, vol. 1, no. 6, pp. 142-145, 1989.

[9] S. Barkou, J. Broeng, and A. Bjarklev, "Dispersion properties of photonic bandgap guiding fibers," in Proceedings of the Optical Fiber Communication Conference, 1999, and the International Conference on Integrated Optics and Optical Fiber Communication (OFC/IOOC '99). Technical Digest, vol. 4, pp. 117-119, 1999.

[10] Z. Várallyay, K. Saitoh, J. Fekete, K. Kakihara, M. Koshiba, and R. Szipocs, "Reversed dispersion slope photonic bandgap fibers for broadband dispersion control in femtosecond fiber lasers," Optics Express, vol. 16, no. 20, pp. 15603-15616, 2008.

[11] J. Riishede, J. Lægsgaard, J. Broeng, and A. Bjarklev, “All-silica photonic bandgap fibre with zero dispersion and a large mode area at $730 \mathrm{~nm}$," Journal of Optics A, vol. 6, no. 7, pp. 667-670, 2004. 

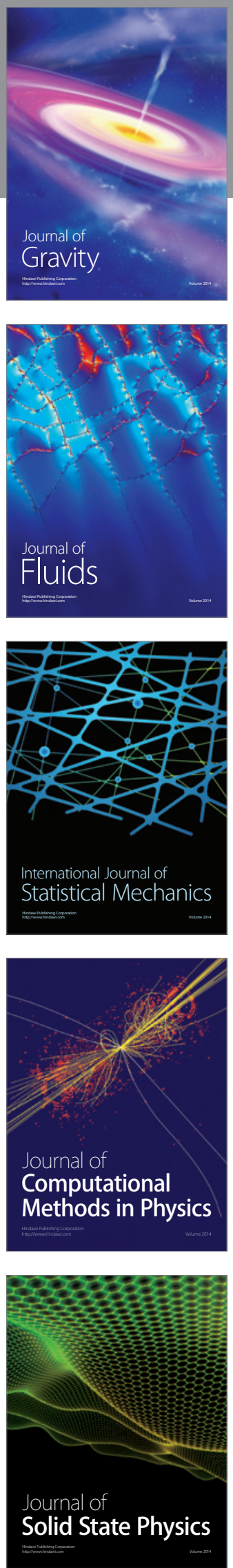

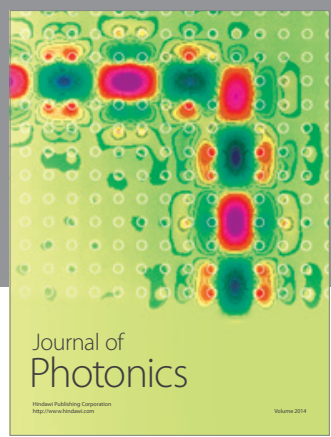

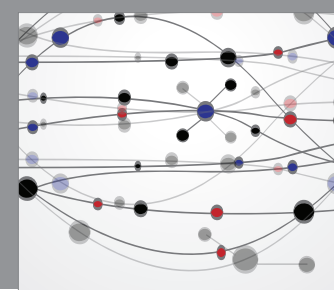

The Scientific World Journal

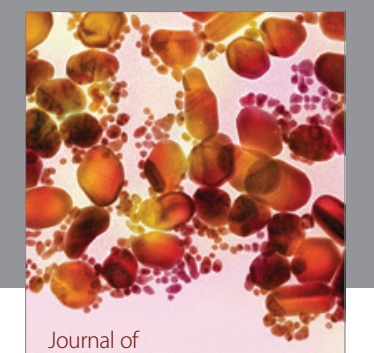

Soft Matter
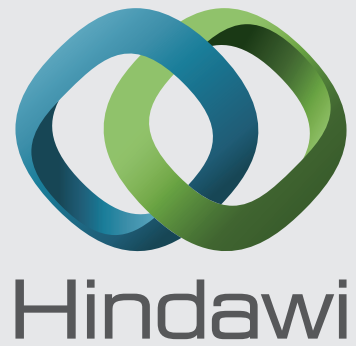

Submit your manuscripts at

http://www.hindawi.com
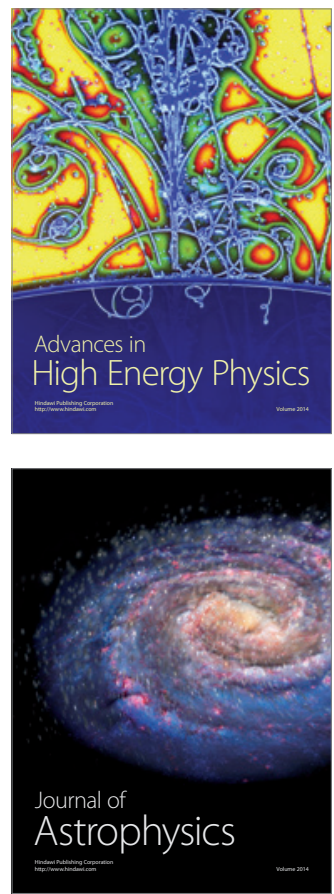
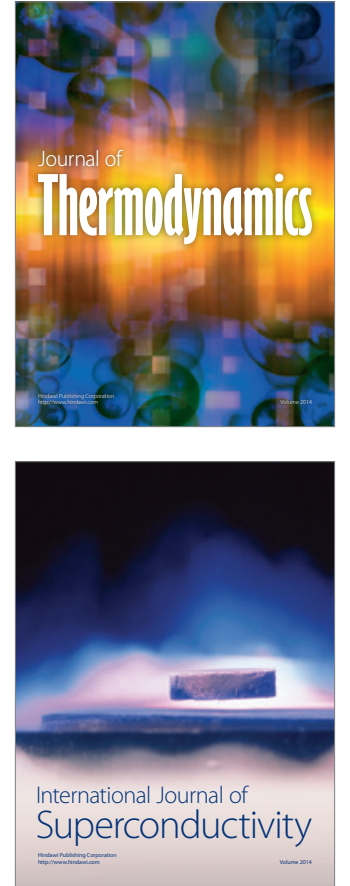
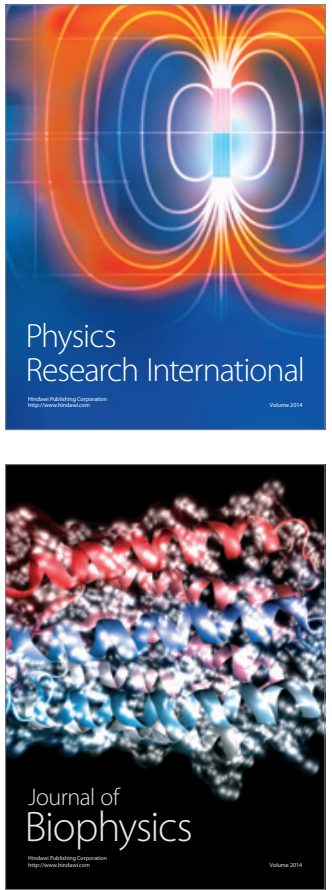
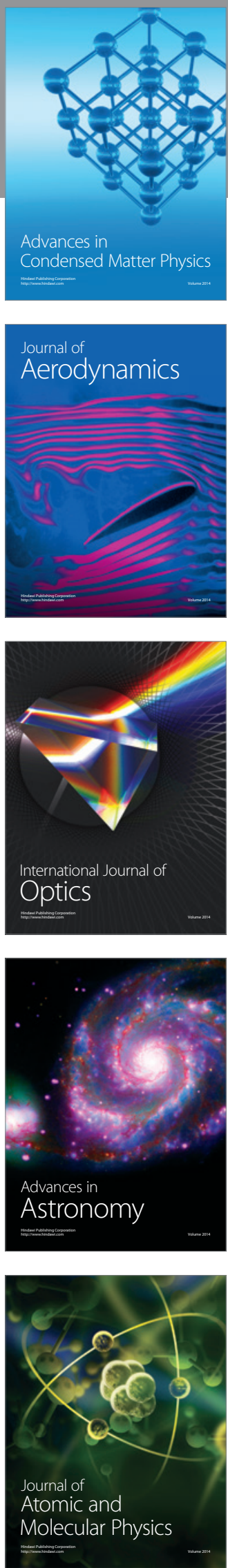